\title{
Family medicine's rapid establishment and early leadership role in Qatar's health care system
}

\author{
This article was published in the following Dove Press journal: \\ Journal of Healthcare Leadership \\ 10 August 2013 \\ Number of times this article has been viewed
}

\author{
Mohamud A Verjee' \\ Mariam Ali Abdulmalik ${ }^{2}$ \\ Michael D Fetters ${ }^{3}$ \\ 'Department of Medical Education, \\ Weill Cornell Medical College in \\ Qatar, Qatar Foundation - Education \\ City, Doha, Qatar; ${ }^{2}$ Primary Health \\ Care Corporation, Doha, Qatar; \\ ${ }^{3}$ Department of Family Medicine, \\ University of Michigan, Michigan, USA
}

Correspondence: Mohamud A Verjee Department of Medical Education, Weill Cornell Medical College in Qatar, Qatar Foundation - Education City, PO Box 24133, Doha, Qatar Tel +97444928366 Fax +974 44928377

Email mov2002@qatar-med.cornell.edu
Abstract: Family medicine is a focus of health care in Qatar, and it has emerged as a primary care pioneer in the Arabian Gulf Region. Strong governmental financial support has underpinned family medicine development in the country, and through proactive health care policy, free or highly affordable health care is available to all citizens and expatriates in primary health care centers and hospitals. An Ivy League and world-class medical school, Weill Cornell Medical College in New York established a second campus in Qatar in 2001, and enrolled its first students in 2002. The inaugural class graduated in 2008, including one graduate who matched to a family medicine residency in the United States. The College has already earned a reputation for an emphasis on cultural sensitivity in the curriculum. Qatar also has a well-established family medicine residency program overseen by the Primary Health Care Corporation. Its inaugural class of family medicine residents began training in 1995 and graduated in 1999. In contrast to a trend of fluctuating interest in family medicine training in many developed countries, the demand for residency slots in Qatar has been consistently high. Since November 2012, the Accreditation Council for Graduate Medical Education-International has approved all hospital-based residency positions. Formed in 2012, the Primary Health Care Corporation is dedicated to achieving accreditation for the family medicine residency in the near future. In 2011, Qatar's 147 family physicians comprised about 18\% of the total physician workforce. Through extended hours of operation at health centers, patients have ready access for acute care and follow-up consultations. Still, Qatar faces challenges including a projected population expansion from about 1.9 million in 2013 to 2.5 million people by 2020. Qatar's National Primary Health Care Strategic Steering Group has recently submitted a new primary health care strategy to the government and identifies 12 challenges for the future of family medicine. Among these, ensuring access to clinical services that are patient and family centered, addressing the shortage of family physicians, expanding academic capacity, and increasing scholarly output are manifest.

Keywords: family medicine, Qatar, undergraduate medical education, residency training, Arabian Gulf Region

\section{Background}

Known historically for pearl diving, fishing, and camel breeding in the 1960s, Qatar is a state in the Arabian Gulf region. Doha, Qatar's capital, has gained distinction with an extremely high density, multicultural, and urban population. ${ }^{1}$ The discovery of massive natural gas fields in the 1960s provided an overnight source of wealth. Qatar's strong leadership is inspired and creative, especially with respect to health care. From the outset, Qatar's leaders envisioned rapid modernization in the arenas of health, education, and academic research. In 2008, Qatar allocated up to 2.5\% of the country's Gross Domestic Product (GDP) to scientific development and research projects. Impressive discoveries, such as the deciphering of the date palm genome ${ }^{2}$ and multiple patent registrations 
are notable. Qatar already has assumed the position as a pioneering role model for development by many Middle Eastern countries of the Arabian Gulf Region. Part of Qatar's development process involves an intensive influx of foreign persons from all over the world arriving on work contracts. The country's health system becomes engaged through mandated health scrutiny of all such arrivals and requires physical examinations, especially for tuberculosis, Hepatitis $\mathrm{B}$, and human immunodeficiency virus (HIV). The prevalence of these diseases ${ }^{3}$ in Qatar is consequently extremely low and closely monitored. In fact, active tuberculosis or confirmed HIV positive status on arrival means repatriation. All the Gulf Cooperation Council countries have a similar program, but Qatar's medical fitness requirements are particularly exacting.

With the Arabian Gulf Region's growing importance, Osman et $\mathrm{al}^{4}$ note the paucity of literature regarding family medicine despite Qatar's emergence as an educational, social, and political leader. Abyad et $\mathrm{al}^{5}$ state that academic family medicine developments in Qatar will have far reaching implications for the specialty in this region given the intense international focus on primary care development and dynamic primary care reform.

The purpose of this paper is to introduce family medicine's dynamic role in health care in Qatar; to provide an overview of the undergraduate medical education system and the role that family medicine faculty play; to illustrate the approach to family medicine training via a 4-year curriculum; and to introduce the central role of family medicine physicians in primary health care centers as key coordinating specialists.

\section{Health care in Qatar}

The Government in Qatar bears all Qatari patients' health expenses incurred at the primary health care center clinics. Expatriates and non-citizens with a Qatar Resident Permit are eligible to attend a primary health care center clinic if they possess a health card, usually subsidized by their employers. Others can purchase a health card for a nominal sum, enabling access to total government health care, including hospital treatment, in Qatar. Care in the private sector provides an alternative for those who have additional private health insurance policies, which have a robust following. The government-led Supreme Council of Health closely monitors these facilities for accreditation.

\section{Undergraduate medical education}

Weill Cornell Medical College New York (WCMC-NY) established a branch campus called Weill Cornell Medical
College in Qatar (WCMC-Q) in 2001. Its establishment was unique after an invitation from the leadership in Qatar in 2000. WCMC-Q is the only medical school in Qatar, and produced its first graduate physicians in May 2008. It has further affiliations with Weill Bugando University College of Health Sciences and Bugando Medical Center in Mwanza, Tanzania ${ }^{6}$ with university student exchange for medical training opportunities from Qatar.

The medical school has a 6-year curriculum: two initial years in premedical and general curriculum are followed by four final years divided between basic medical sciences (for 2 years), and clinical clerkships and electives (for 2 years). Students are selected from both Qatari nationals and international students after interviews and based on academic merit. The college's admission panel is comprised of members from premedical and clinical faculty, and is headed by an executive committee. A 1-year premedical preparatory program exists for students who may have experienced less rigorous science training during high school. Medical school enrollment at WCMC-Q began in 2002 with 16 students per year, and rose to 44 per year in the period between 2008-2012. While all students are fluent in English, approximately $65 \%$ also speak or comprehend Arabic. Uniquely, medical interpreters speaking Arabic, Urdu, Hindi, Malayalam, Telugu, Pashto, and Tagalog are provided for medical students. These interpreters are available to attend or remain on site at WCMC-Q teaching centers, in order to help students communicate with patients. ${ }^{1}$

The curricula of both WCMC-NY and WCMC-Q flow in parallel, with rigorous assessment and evaluation methods and performance expectations. Visiting faculty, live video lecture presentations, and exchanged resources from WCMCNY, provide the academic support required for consistency of curriculum delivery. Premedical faculty teach basic sciences for up to 58 students in the 1st and 2nd premedical years. Medical faculty, qualified as MDs or PhDs, currently teach the longitudinal curriculum for up to 44 students per class in the subsequent four MD medical years. There are two onsite family medicine faculty at WCMC-Q and 17 clinical faculty in the 23 primary health care center clinics.

Cultural sensitivity has been a priority since the opening of the Center for Cultural Competence in Health Care at WCMC-Q in 2011. ${ }^{1}$ The Center plays an important role in addressing cultural differences and diversity, an area of importance as illustrated by Fins and del Pozo, ${ }^{7}$ who report that the medical curriculum is influenced by the hidden curriculum (in different cultural contexts) and on how it impacts communication during educational years. 
The family medicine/primary care clerkships at WCMC-Q last 6 weeks and are scheduled during the 3rd and 4th years of the MD course program. Four weeks are allocated to two clinical faculty family physician preceptors in a primary health care center clinic (Table 1). Another week is allocated to ambulatory care in an emergency department at $\mathrm{Al}$ Khor Hospital, Al Khor, Qatar, under the supervision of two consultant emergency physicians. One further week in sports medicine takes place at Aspetar Orthopedic Hospital in Doha, Qatar. Students rotate through specialized education in outpatient medicine, athletic screening, radiology, and physiotherapy with rehabilitation clinics. Sports medicine electives have proven popular with both domestic and international medical students. Academic outcomes at both teaching sites in New York (WCMC-NY) and Doha (WCMC-Q) show good correlation.

To date, medical school graduates who have chosen family medicine as a career have pursued residencies in Qatar or in the United States. For example, six WCMC-Q graduates have matched to US family medicine residencies since 2008 , one each in Wyoming, Virginia, and Arizona, and three in Connecticut. Two have achieved chief resident status. Graduates with Qatari ties have the option to stay for residency training in Qatar; this means that international graduates seeking to match to family medicine residencies in Qatar face strong competition.

\section{Family medicine residency training}

Family medicine residency training in Qatar lasts 4 years and has a rigorous curriculum including training and evaluation. Year one includes rotations in community medicine, internal medicine, cardiology, and family medicine; year two involves rotations in pediatrics, general and orthopedic surgery, urology, and obstetrics and gynecology; year three includes rotations in psychiatry, emergency medicine, dermatology, ear, nose and throat (ENT), ophthalmology, radiology, and laboratory work; year four rotations involve an opportunity to observe nurse-led health care in schools, a refresher in community medicine, 3 months in electives, and 8 months in family medicine. Residents return to a designated primary

Table I Certified WCMC-Q academic teaching health centers in Qatar in March 2013

\begin{tabular}{ll}
\hline Names of teaching health centers & $\begin{array}{l}\text { Number of accredited } \\
\text { clinical faculty }\end{array}$ \\
\hline West Bay Health Center & 3 \\
Airport Health Center & 3 \\
Umm Ghwalina Health Center & 2 \\
Gharrafa Health Center & 2 \\
Mesaimeer Health Center & 2 \\
\hline
\end{tabular}

Abbreviation: WCMC-Q, Weill Cornell Medical College in Qatar. health care center clinic for 48 half days per year, in each of the first 3 years, and part of the fourth. Up to 1 month's vacation can be taken throughout each training year.

Procedural training receives increasingly heavy emphasis in Qatar. Enhancing current family physicians' procedural abilities, such as casting, skin biopsies, minor surgery for sebaceous cyst excision, and endometrial biopsies should prove to be more cost effective than falling back on expensive hospital referrals. Nothnagle et $\mathrm{al}^{8}$ clearly articulate the current spread and variance of procedural skills implementation in the United States. The focus on augmenting the scope of procedural skills during residency training may also provide enhanced professional satisfaction during and after training.

Local graduate residents are eligible for employment after successfully passing the Family Medicine Arab Board Examination. This is a two-part assessment consisting of a written paper and a combined oral and Objective Structured Clinical Examination (OSCE). Globally recognized residency programs, usually from the US, Canada, or the United Kingdom, also enable eligibility for employment without additionally taking the Family Medicine Arab Board Examination. Qatar's family medicine residency program receives applications from all areas in the Middle East and North Africa, and ranks high among specialties competed for (Table 2), a trend contrasting with fluctuating interest in developed countries.

\section{International medical graduates}

International medical graduates with appropriate qualifications are eligible for employment as physicians but have to meet the Qatar Supreme Council for Health requirements for licensure. All medical qualifications achieved by applicants are carefully vetted before ratification. The Supreme Council for Health issues annual medical licenses after obligatory HIV test clearance and periodic chest X-ray assessment. Proof of Hepatitis B immunity by vaccination or by testing for hepatitis B surface antibody titers is also a requirement for all practicing doctors.

The development of a high quality, globally recognized family medicine training program in Qatar is the foundation for the Qatar National Vision 2030 plan. ${ }^{9}$ The Primary Health Care Corporation currently needs to meet the standards of the Accreditation Council for Graduate Medical Education - International (ACGME-I), an international arm of the ACGME in the United States. ${ }^{10}$ Until 2012, Hamad Medical Corporation was the only government management and operational authority for primary care in Qatar. The Primary Health Care Corporation was devolved from it in 
Table 2 Overview of the family medicine residency program in Qatar from 1995 to March 2013

\begin{tabular}{lc}
\hline Parameters & \\
\hline Success rate of graduation from program & $>95 \%$ \\
Numbers of graduates produced & 90 \\
Applications for FM residencies per annum & -400 \\
Number of FM residencies completed in 2010 & 22 \\
Number of senior consultants & 16 \\
Number of consultants (equivalent of attending) & 34 \\
Number of FM specialists & 43 \\
Total number of residents in FM training (current) & 48 \\
Number of core training faculty & 12 \\
Number of assisting training faculty & 18 \\
Total training faculty & 30 \\
\hline
\end{tabular}

Abbreviation: FM, family medicine.

2012 and is now responsible for all national family medicine administration and operational management. Hamad Medical Corporation passed accreditation for ACGME-I in November 2012. Hence, Qatar is the first country in the Middle East to be accredited and, along with Singapore, is one of only two countries outside the United States to have achieved this accreditation (ACGME-I), since 2009. In the Gulf Region, the United Arab Emirates with six health units, Oman with another two, and Kuwait with one, are now seeking ACGMEI accreditation. The Primary Health Care Corporation aims to achieve this same accreditation for its family medicine residency by the year 2014 .

\section{Family medicine's rapidly emerging role in Qatar's health care system}

In 1978, Qatar established a primary health care system and 17 years later recognized family medicine as a specialty in 1995 (Table 3). Launched in 2012, the newly formed Primary Health Care Corporation prioritizes prenatal, childcare, and well women clinics in addition to providing general care. The full complement of health care staff include family physicians, one of whom serves as the director, nurses (medical and dental), pharmacists, dispensers, radiographers, physiotherapists, counselors, administrators, receptionists, security, and support staff. Primary health care center clinics also use an in-house model of specialty consultation with access to otolaryngology, ophthalmology, cardiology, obstetrics, gynecology, dermatology, oral hygiene, and dental care. Chronic diseases, such as diabetes, asthma, hypertension, and geriatric programs are incorporated into the family medicine setting (Table 4). In 2011, Qatar employed 147 family physicians, or 18\% of its total physician workforce, including salaried family medicine residents. Residents must take the local Arab
Board qualification in family medicine, an oral examination conducted in English, to complete the final step in training. Patient attendance at primary health care centers is robust with hours of operation on a shift system from 7:30 am to 11 pm daily in a number of health center sites. Patients now receive confirmation of any family physician referrals to specialists, known locally as consultants, via mobile phone SMS text messages, a service enhancing efficiency and improving communication.

While Qatar has faced challenges in providing equitable delivery of health care in the past, the Primary Health Care Corporation now has autonomy to develop its own organizational infrastructure. Major changes are afoot with Qatar's Primary Health Care Strategic Steering Group, an advisory panel created to produce a National Primary Health Care strategy for the Government by January 2013. The Right Honorable Lord Ara Darzi, a former British Minister of Health previously involved in primary health care reform in the United Kingdom, integrally co-chaired a planning team with one of the authors (MA); their efforts will contribute to significant advances in health care. ${ }^{11}$ Moving forward rapidly, Qatar is among the leaders in

Table 3 Selected characteristics of family medicine in Qatar in 2012

\begin{tabular}{ll}
\hline Characteristics & Qualifying description \\
\hline Family medicine recognized as & 1995 \\
specialty in Qatar & \\
Family physicians employed & 147 \\
in PHCCs in Qatar & \\
$\quad$ Senior consultants & 16 \\
Consultants & 34 \\
Specialists & 14 \\
Other advisors & 6 \\
Clinical faculty members & 17 in the departments of family \\
& medicine, PHCCs \\
Medical school & Weill Cornell Medical College \\
& in Qatar \\
Medical students training per year & $40-44$ \\
Medical clerkship duration & 6 weeks: four in FM, one in \\
& emergency medicine, one in \\
Fength of FM residency program & 4 years \\
FM residency training places & 48 (distributed between 4 years) \\
Medical clerkship placement in health & 4 weeks \\
centers & 23 \\
FM residents in training at PHCCs & $10-13$ per year \\
Total FM residents in training & 18 \\
Male & 30 \\
Female &
\end{tabular}


Table 4 Scope of service in primary health care centers in Qatar

\begin{tabular}{l} 
Services \\
Ambulatory care \\
Chronic disease care clinics \\
Counseling and social/family guidance/marital counseling \\
Dental care for all patients \\
Diabetic foot ulcer clinic \\
Dressing changes \\
Emergency care provided by HMC staff affiliated with health care \\
centers \\
Intravenous therapy/injections \\
Laboratory: venesection, patient sample processing, stat results, reports \\
Minor operational/surgical procedures (selected) \\
Pediatric surveillance \\
Pharmacy: online formulary with HMC for recording and consistency of \\
prescriptions \\
Prenatal care \\
Preventive care (screening, such as premarital counseling, \\
immunization, etc) \\
Postpartum care \\
Resident teaching for family medicine (selected) \\
Sterile supply and instrument sterilization \\
Vaccinations and preventive care \\
Visiting consultant clinics, eg, dermatology, ophthalmology \\
Well baby clinic \\
Well women clinic \\
X-ray with ultrasound (selected) \\
\hline
\end{tabular}

Notes: Academic teaching health centers for PHCC family medicine residents; up to I2 selected. Academic teaching health centers for WCMC-Q; five in total from July 2012. Abbreviations: HMC, Hamad Medical Corporation; PHCC, primary health care centers; WCMC-Q, Weill Cornell Medical College in Qatar.

family medicine in the Arabian Gulf, Middle East, and North African region. The challenges of building a new family physician workforce and overcoming specialty care misperceptions are much the same as any other emerging country from the 1990s. Qatar is unique due to its dedication in developing a full and modern health care system from the outset, grounded in family medicine, and its active planning for a comparatively small but growing population, currently polled at 1.9 million. $^{12}$

\section{Challenges for the future}

The Qatar Statistics Authority (2013) ${ }^{12}$ projects the country's population will rise from 1.9 million to over 2.5 million by 2020. Qatar's visionary primary health care leaders are careful with demographic planning. All health care providers and patients will benefit from the implementation of a uniform, national health electronic medical record system. A portable smart card medical record is foreseen, with access controlled by the patient or other authorized user such as a parent, guardian or, for emergency use, by qualified professionals.
Embracing this rapid population growth, family medicine in Qatar faces challenges that include:

1. Acquiring knowledge of local disease patterns to plan for future needs.

2. Expanding the scope of practice by expanding procedural skills training.

3. Incorporating the latest suitable information technology.

4. Providing best practice guidelines and protocols for modern health screening.

5. Obtaining coherent feedback on the residency program, addressing needs and assessing educational outcomes from validated evaluations, eg, by using formative and summative Objective Structured Clinical Examinations (OSCEs). ${ }^{13}$

6. Enhancing a global standard of resident family medicine certification by achieving ACGME-I accreditation in family medicine rather than being restricted to Arab Board certification as at present. ${ }^{4}$

7. Increasing residency output as capacity permits.

8. Providing employment opportunities for new family medicine graduates in established primary health care centers in Doha, as well as serving outlying rural communities in Qatar.

9. Actively addressing one of the most important challenges Qatar encounters, namely the shortage of family physicians, especially Qatari.

10. Tracking continuous professional development and continuous medical education for all employed family physicians annually.

11. Striving for scholarship within the academic primary health care centers as exemplified by peer reviewed publications, presentations at international conferences, and academic or service recognition and promotion.

12. Mentoring and supporting new graduate family physicians by fully qualified and established primary care physicians. ${ }^{14}$

\section{Conclusion}

As change at an extremely rapid pace is the modus operandi in Qatar, further significant evolution of the primary health care system is likely. Establishing primary health care centers with family physicians in leadership roles is of paramount importance for success. Construction of new primary health care centers is in the offing, and will help achieve the vision of Qatar's National Health Strategy. Given the pace of development of family medicine internationally and its rapid growth and demand in Qatar, its physicians will need to strive to meet these challenges, not 
only with service but with increased academic ability and scholarly output.

\section{Acknowledgments}

The authors obtained confirmation from the Office of Research Compliance at Weill Medical College in Qatar, that this paper does not describe research with human subjects as defined by applicable regulations. No ethical review was therefore required. The authors thank Beth Ragle, Administrative Assistant in the Department of Family Medicine, University of Michigan, Ann Arbor, MI, United States, for assistance in the preparation of this paper.

\section{Disclosure}

The authors report no conflicts of interest in this work.

\section{References}

1. Elnashar M, Abdelrahim H, Fetters MD. Cultural competence springs up in the desert: the story of the center for cultural competence in health care at Weill Cornell Medical College in Qatar. Acad Med. 2012;87(6):759-766.

2. Al-Dous EK, George B, Al-Mahmoud ME, et al. De novo genome sequencing and comparative genomics of date palm (Phoenix dactylifera). Nat Biotechnol. 2011;29(6):521-527.

3. www.sch.gov.qa [homepage on the Internet]. Emergency and Disease Control Section. Supreme Council for Health, Qatar; 2012. Available from: http://www.sch.gov.qa/sch/En/scontent.jsp?smenuId=70. Accessed February 28, 2013.

4. Osman H, Romani M, Hlais S. Family medicine in Arab countries. Fam Med. 2011;43(1):37-42.

5. Abyad A, Al-Baho AK, Unluoglu I, Tarawneh M, Al Hilfy TK. Development of family medicine in the middle East. Fam Med. 2007;39(10):736-741.
6. www.weill.cornell.edu [homepage on the Internet]. Weill Cornell Bugando Program. Weill Bugando University College of Health Sciences; 2011. Available from: http://weill.cornell.edu/globalhealth/ majorinitiatives/weill_cornel1_program_in_bugando.Accessed September 21, 2012.

7. Fins JJ, Rodríguez del Pozo P. The hidden and implicit curricula in cultural context: new insights from Doha and New York. Acad Med. 2011;86(3):321-325.

8. Nothnagle M, Sicilia JM, Forman S, et al; STFM Group on Hospital Medicine and Procedural Training. Required procedural training in family medicine residency: a consensus statement. Fam Med. 2008;40(4): 248-252.

9. General Secretariat For Development Planning. Qatar National Vision Document 2030. Doha: General Secretariat For Development Planning; 2008. Available from: http://www.gsdp.gov.qa/portal/page/ portal/gsdp_en/qatar_national_vision/qnv_2030_document/QNV2030_ English_v2.pdf. Accessed December 15, 2012.

10. ACGME. ACGME-International Advanced Specialty Program Requirements for Graduate Medical Education in Family Medicine. City: Publisher, 2012. Available from: http://www.acgme-i.org/web/requirements/ familymedicine.pdf. Accessed November 10, 2012.

11. Department of Health. High Quality Health for All, NHS Next Stage Review Final Report. Department of Health; 2008. Available from: http://www.official-documents.gov.uk/document/cm74/7432/7432.pdf. Accessed April 24, 2013.

12. www.qsa.gov.qa [homepage on the Internet]. Our Census, Our Future. Qatar Statistics Authority; 2010. Available from: http://www.qsa.gov. qa/QatarCensus. Accessed February 28, 2013.

13. Alinier G. Nursing Students' and lecturers' perspectives of objective structured clinical examination incorporating simulation. Nurse Educ Today. 2003;23(6):419-426.

14. Young RA, DeHaven MJ, Passmore C, Baumer JG, Smith KV. Research funding and mentoring in family medicine residencies. Fam Med. 2007; 39(6):410-418.
Journal of Healthcare Leadership

\section{Publish your work in this journal}

The Journal of Healthcare Leadership is an international, peer-reviewed, open access journal focusing on leadership for the health profession. The journal is committed to the rapid publication of research focusing on but not limited to: Healthcare policy and law; Theoretical and practical aspects healthcare delivery; Interactions between healthcare and society and evidence-based practices;

\section{Dovepress}

Interdisciplinary decision-making; Philosophical and ethical issues; Hazard management; Research and opinion for health leadership; Leadership assessment. The manuscript management system is completely online and includes a very quick and fair peer-review system. Visit http://www.dovepress.com/ testimonials.php to read real quotes from published authors. 